University of Montana

ScholarWorks at University of Montana

Graduate Student Theses, Dissertations, \&

Professional Papers

1953

\title{
Pinacol reduction of ketones at plated copper gauze cathodes
}

William Joseph Sullivan

The University of Montana

Follow this and additional works at: https://scholarworks.umt.edu/etd

Let us know how access to this document benefits you.

\section{Recommended Citation}

Sullivan, William Joseph, "Pinacol reduction of ketones at plated copper gauze cathodes" (1953).

Graduate Student Theses, Dissertations, \& Professional Papers. 8178.

https://scholarworks.umt.edu/etd/8178

This Thesis is brought to you for free and open access by the Graduate School at ScholarWorks at University of Montana. It has been accepted for inclusion in Graduate Student Theses, Dissertations, \& Professional Papers by an authorized administrator of ScholarWorks at University of Montana. For more information, please contact

scholarworks@mso.umt.edu. 
THE FIriCOL REDUCTIN CF KUTONES AT FLATED

\author{
COEPER GEULE CATHODNS \\ BY \\ :ILIIAU JOSTII SULIIVIN
}

B. Á.

BONTANA STATT UUIVIRSTT, 1951

B. S. In Tharmacy WOLTHA STATS UITERSTY, 1952

Fresented in fartial fulfillaent of the

requirerents for the degree of

Liaster of Arts

SONTAHA STATE UIJIVARSTTY

1953

Approved by:

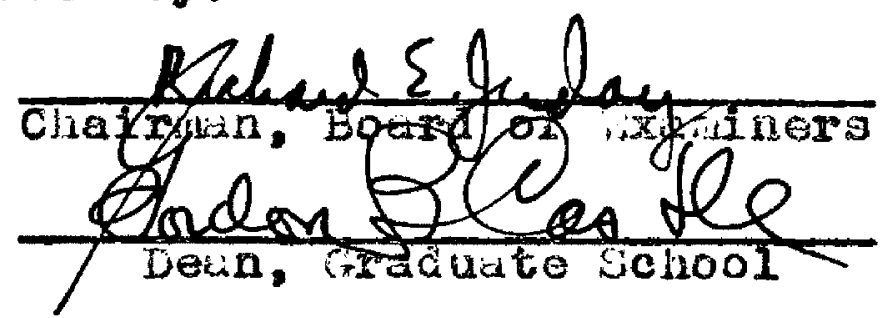

May, $\frac{29,19.3}{\operatorname{set} 0}$ 
All rights reserved

\section{INFORMATION TO ALL USERS}

The quality of this reproduction is dependent upon the quality of the copy submitted.

In the unlikely event that the author did not send a complete manuscript and there are missing pages, these will be noted. Also, if material had to be removed, a note will indicate the deletion.

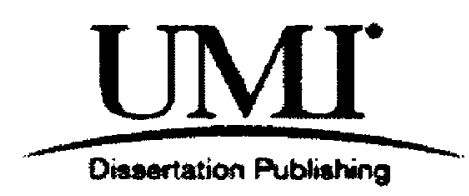

UMI EP38979

Published by ProQuest LLC (2013). Copyright in the Dissertation held by the Author.

Microform Edition (C) ProQuest LLC.

All rights reserved. This work is protected against unauthorized copying under Title 17. United States Code

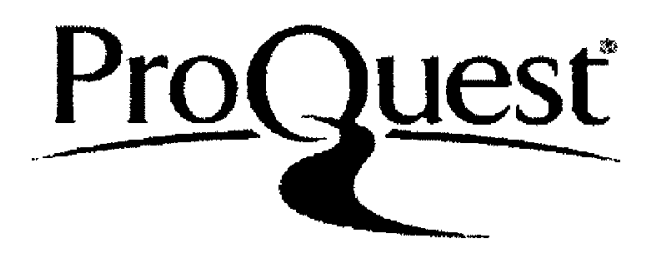

ProQuest LLC.

789 East Eisenhower Parkway

P.O. Box 1346

Ann Arbor, MI 48106 - 1346 
ACKWO LEDG:TENS

The euthor is deeply indebted and

especially gratoful to Dr. R. E. Juday for

his competant guldance throughout the project. 
MABLE Or COUTLATS

$F \cdot \operatorname{sig}$

INTRODUCTICN

The Froblan . . . . . . . . . 1

Hethod of Study . . . . . . . . 2

DISCUSSICN

Cathodes . . . . . . . . . 4

Tabular Summary of Results . . . 5

hodiun ............ I4

Temperature . . . . . . . . 15

Current Density . . . . . . . 16

furificstion of pinacol ..... 16

Proof of structure ... . . . . 17

Summary ............. . 19

Flow Sheet, Figure I. . . . . . 20

E.PERI:TPTAL

pparetus, El gure II . . . . . . 21

Reduction . . . . . . . . . $2 t_{s}$

Eurification of the froduct . . . $2 B$

Preparation of Derivatives . . . 29

LITHATURE CITED • . . . . . . . 32 


\section{INTRODUCTION}

Amalgams of active metels such as aluminum and magnesium have been the reducing agents used most for converting ketones to the corresponding pinacols. This technique suffers from serlous disadvantages, however, such es the special preparations of the amalgat, use of large volumes of solvent, and the formation of large quantities of flocculent precipitates which interfere $w^{*}$ th the isolat1on of the product. Reduction of the ketone at the cathode of an electrolytic cell avolds these difriculties and is especially adepted for large scale operations. The principal problem that arises in the use of this technicue is to obti in a cathode which will give high current erficlenoles, 1. e. produce little hydrogen.

The rroblem.- The electrodes used nost have been made of the metal, cast or rolled into the proper shape, with or without a plating of another metal. It is, however, rrequently inconvenient to make this type of electrode in the absence of metallurgical erulpment and commercialy arellable sheet metale frequently contain small amounts of impurities which interfere with the reduction. The purpose of this study was to develop a cathode daterial which was readily avallable, and would produce the desired 
reduction in good gield with high current efficlency. The material selected was cofper gauze. Besides its avallability and purity, copper gauze has the additional advantages of being $11 \mathrm{ght}$, easily shaped into the desired form, and readily plated with other metels. Copper gauze, both plain and plated, has been used as an electrode in the reduction of various substances, la,b but no gysteratic study has been mado of it as the cathode for tho reduction of elther ketones or any other class of compounds. Tho overvoltage of pure copper is too low to reduce the ketone by itself, but by plating the copper with a metal of tieh overvoltage, it has been found that the cathode behaves as if made of that material oven though the plating is so thin as to be virtually invisible.

$$
\text { lethod of study.- P-hydroxyproplopienone mas }
$$

selected as the ketone and its reduction was gtudied varying the cathode, pH, temperature, and curront density. Besldes being comercially avallable and relatively inexpensive, this compound has the adrantage of belne soluble In aqueous alkall and since it is an unsymmetrical ketone, 1t cun form two isomeric pinacols so that the ratio of isomers formed under various conditions can also be stualed. other workers have reported the electrolytic roduction of this ketone at a load cathode, 2 but no detailod study has been made and the yields of pinacol have been low. The apparatus used was simflar to thet previously described.3 The metals selected for plating woro ones which were expected 
to have a high overvoltage so as to bring about the desired reaction. No really accurate method for deternination of overvoltage has been developed, however, it is possible to make qualitative comparisons and on the basis of the work of Casparit and Tafel, 5 lead, mercury, tin, cadmium, thallium, arsenic, antimony, and bismuth were selected. The results obteined are summarized in Table I. The yields reported are the total crude yield, contalning a mixture of the dl and meso isomers after the senaration of gumny materials and unreacted starting material. This was felt to give the best idea of actual yield obtained since soparation of the isomers entalled some handinf losses. The process was also too tedious to be practical for individual runs.

\section{DISCYSETON}

ihen using cathodes of 40 and 60 mesh, the current erriciency depended on the plating netal used. In the case of 20 mesh gauze and copper tre of 26 gauge the current efflclenoy was very low in all cases, even wen pilated with metals which gave high currert efficiencias vith the finor resh. Since it was inconvenient to serarite unreacted starting material, an excess of current was 
generally passed through the cell. ihen the amount of current used wis ereatiy in excess of that needed, for the formation of the pinacol in acid medium, no attack on the plnacol was noted. However, whon an excesa of current was used in alkaline medium there was furthex reduction of the pinecol giving rise to elumy products. Consequently, the amount of current used in alkaline modiun was just slightly in excess of the theoretical. Cothodes.- Mhs metals used in pluting tho copper gauze and the coatings obtained were critlcal. It was subs that in the case of mercury, as little as $6 \mathrm{mg}$. of mercury over a surface area of $330 \mathrm{sq}$. cm. efrected a reduction in acid medium, whereas, a heavily mercury plated cathode give a smaller yield of pinacol. It was also noted that the cathode orleinally plated with 6 ag. of mercury could be used in two more such reactions before it was necessary to replate the cathode. When this replating was found necessary $1.5 \mathrm{ag}$. of mercury reproduced the original activity. In alkaline medium, the heavily mercury plated cethode wes found most efficlent. lost probably a sodiun alligam was formed, for when a pool of mercury was used as the cathode, excellent results were obtained, but the pool beesn to solidify and when washed with water, a steady ovolution of hydrogen was noted. Copper gauze was also hoavily plated with nickel and silver and these were thon amalgamated. The reaults obtained were poorer than for analganated conper. 


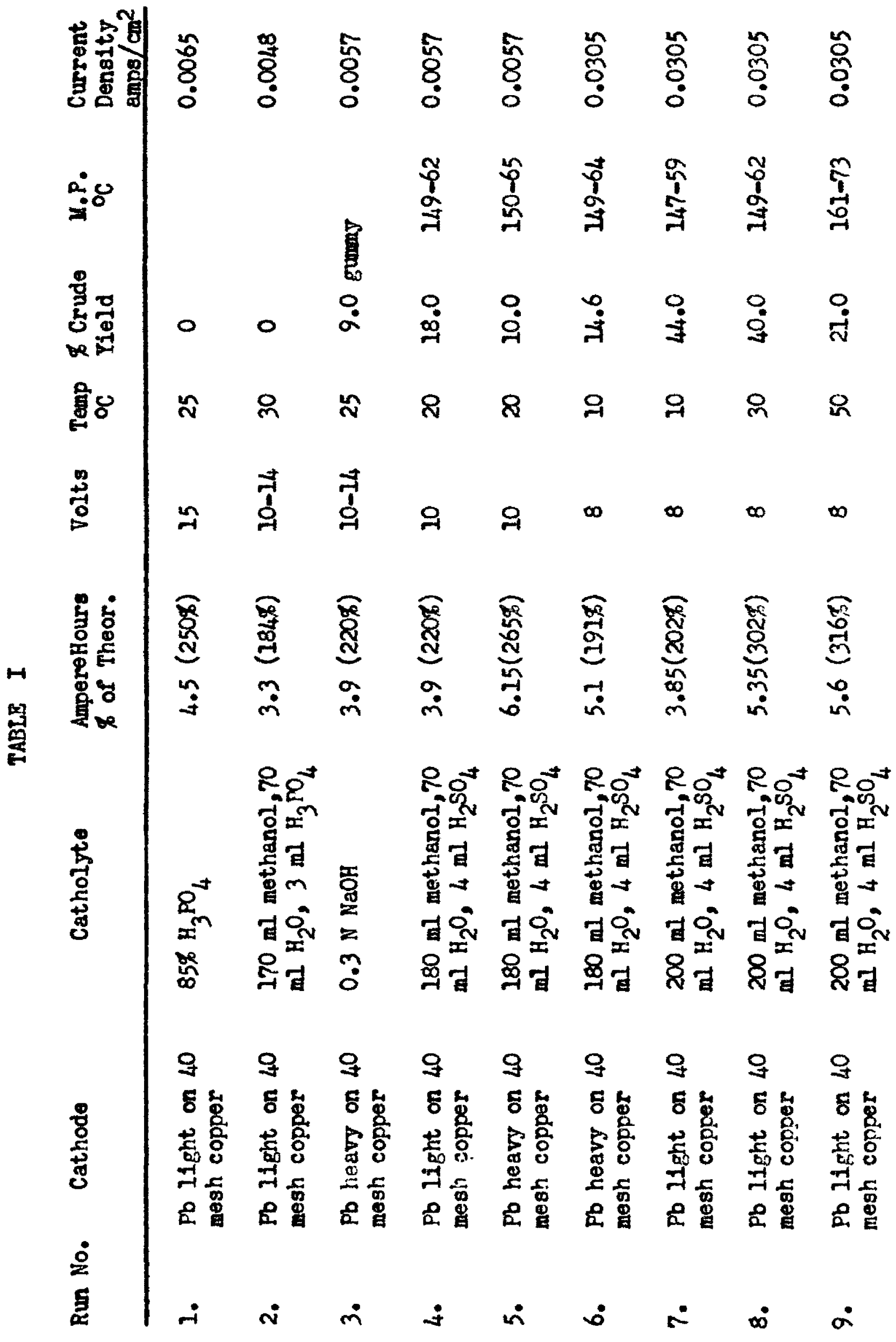


$\frac{\text { Run No. Cathode }}{\text { 10. Pb light on } 40}$ (Reused \# 9)

11. Pb 11ght on 40 mesh copper

12. Pb light on 40 mesh copper

13. Fo Iight on 40 mesh copper

14. Pb 11ght on 60 mesh copper

15. $\mathrm{Pb}$ light on 60 mesh copper (Reused "14)

16. $\mathrm{Pb}$ 11ght on 60 mesh copper

17. Pb light on 60 mesh copper

18. $\mathrm{Pb}$ light on 60 mesh copper No Diaphragm.

19. Pb light on 60 mesh copper

Catholyte

Ampero Hours

\& of Theor.

Volts

Temp $\$$ Crude $H_{0} P$.
${ }^{\circ} \mathrm{C}$ Yleld

Current Density,

200 mil mathanol,70 ml $\mathrm{H}_{2} \mathrm{O}, 4 \mathrm{ml} \mathrm{H}_{2} \mathrm{SO}_{4}$

$5.6(3168)$

8

25-40

43.0

$160-170$

0.0305

$200 \mathrm{ml}$ methanol,70

ml $\mathrm{H}_{2} \mathrm{O}, 4 \mathrm{ml} \mathrm{H}_{2} \mathrm{SO}_{4}$

$200 \mathrm{ml}$ methanol, 70 ml $\mathrm{H}_{2} \mathrm{O}, 4 \mathrm{ml} \mathrm{H}_{2} \mathrm{SO}_{4}$

$200 \mathrm{mil}$ methanol, 70 ml $\mathrm{H}_{2} \mathrm{O}, 4 \mathrm{ml} \mathrm{H} \mathrm{SO}_{4}$

$125 \mathrm{ml}$ methanol, 35 ml $\mathrm{H}_{2} \mathrm{O}, 2 \mathrm{ml} \mathrm{H}_{2} \mathrm{SO}_{4}$

$125 \mathrm{ml}$ methanol, 35 $\mathrm{mI} \mathrm{H}_{2} \mathrm{O}, 2 \mathrm{ml} \mathrm{H}_{2} \mathrm{SO}_{4}$

$4.25(300 \%)$

$4.9(2768)$

$6.3(350 \%) \quad 10$

$2.2(21,0 \%)$

7.5

$2.2(240 \%)$

7.5

25

32.0

146-148 0.0063

St. Mat.

125 ml methanol, 35 ml $\mathrm{H}_{2} \mathrm{O}, 3 \mathrm{ml} \mathrm{H}_{2} \mathrm{SO}_{4}$

$125 \mathrm{ml}$ methanol, 35 ml $\mathrm{H}_{2} \mathrm{O}, \mathrm{I} \mathrm{ml} \mathrm{H}_{2} \mathrm{SO}_{4}$

$125 \mathrm{ml}$ methanol, 35 ml $\mathrm{H}_{2} \mathrm{O}, 2 \mathrm{ml} \mathrm{H} \mathrm{SO}_{4}$

$3.3(360 \%) \quad 7-30 \quad 30 \quad 0$

0.0063

$125 \mathrm{ml}$ methanol, 35 ml $\mathrm{H}_{2} \mathrm{O}, 5 \mathrm{ml} \mathrm{H}_{2} \mathrm{SO}_{4}$

30

18.0

$144-47 \quad 0.0063$ 


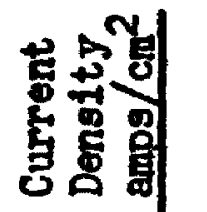

๕్

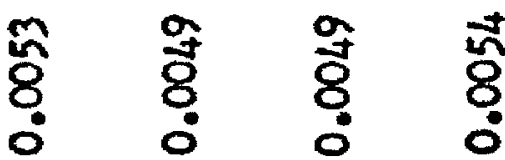

雚

คi:

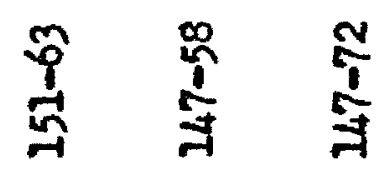

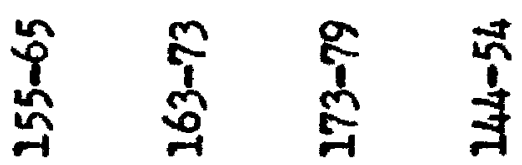

蒂

$\underset{3}{9} \frac{9}{4}$

$\begin{array}{llll}0 & 0 & 0 & \\ \infty & 0 & 0 & 0\end{array}$

$\begin{array}{llll}0 & 0 & 0 & 0 \\ 0 & \sqrt{0} & 8 & 0\end{array}$

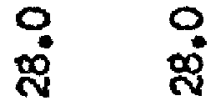

量乐

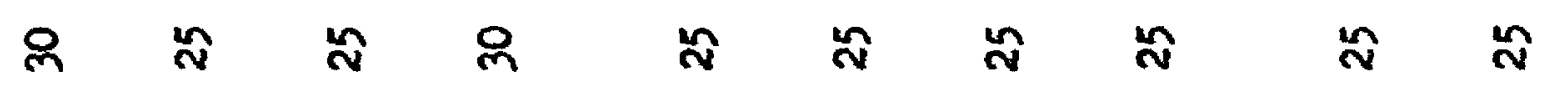

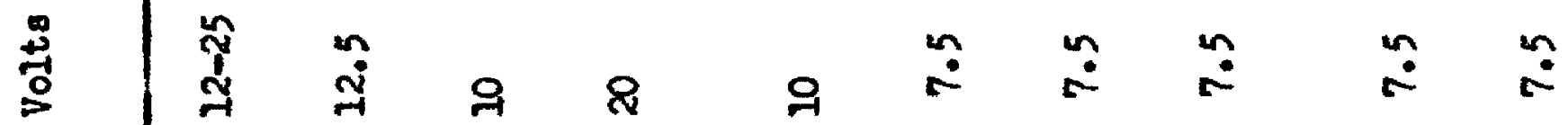

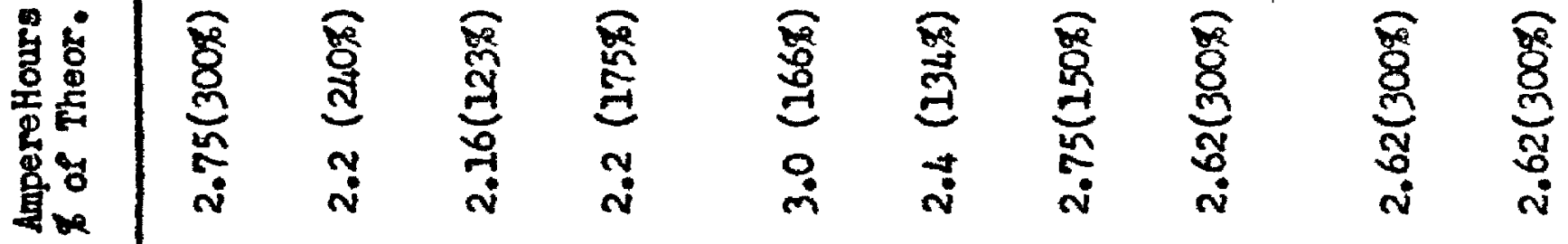

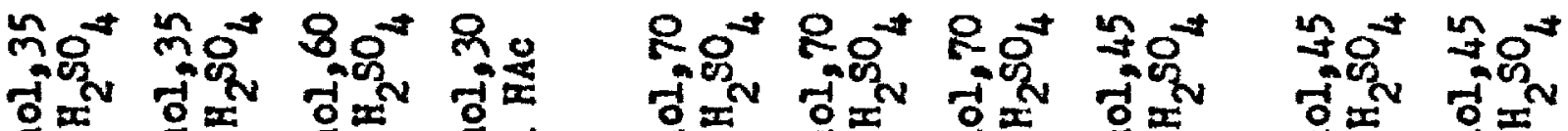

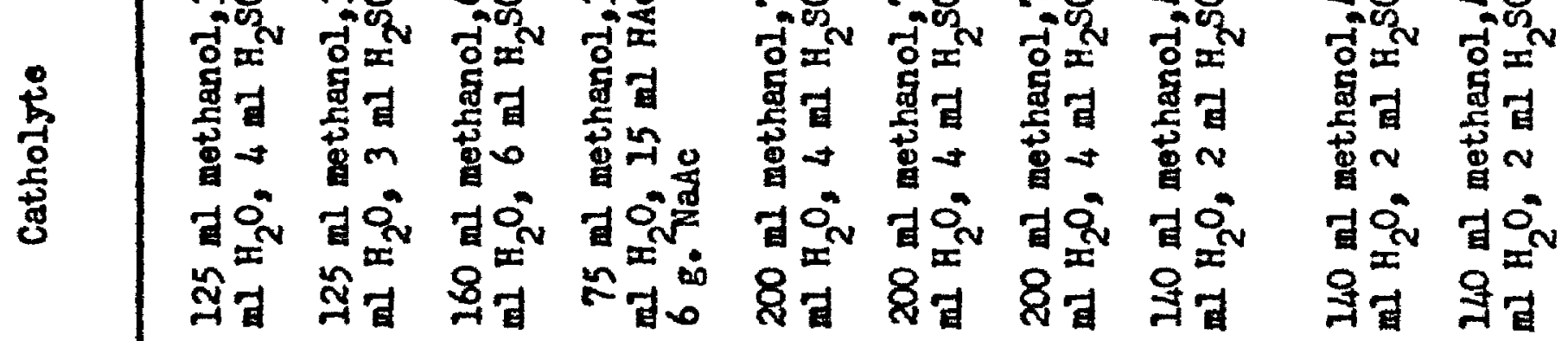

8888

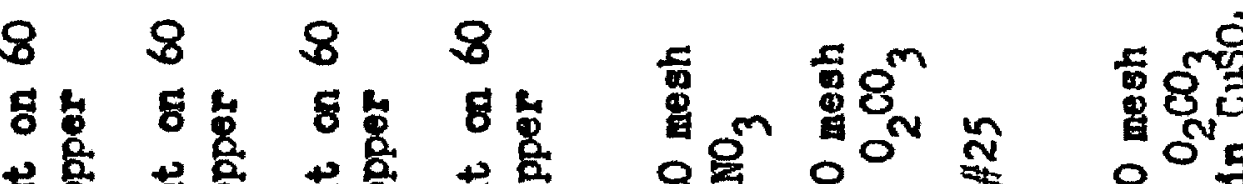

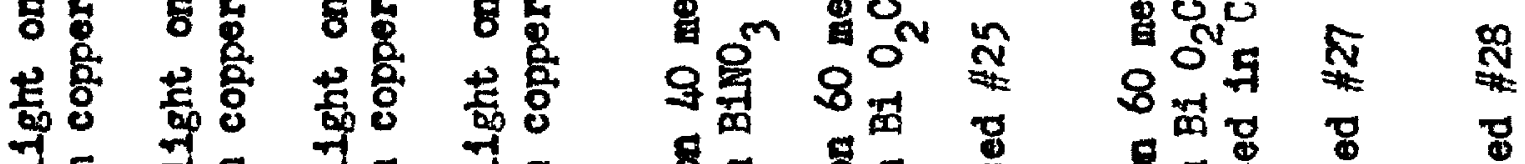

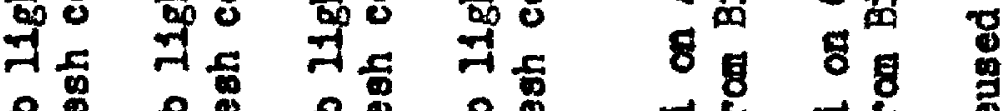

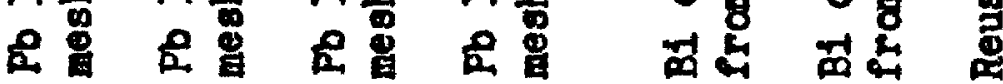

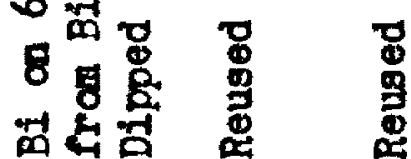

$\stackrel{\circ}{2}$

동

8

X

พิ

त)

กิ่

5

$\stackrel{\infty}{8}$ 
韵

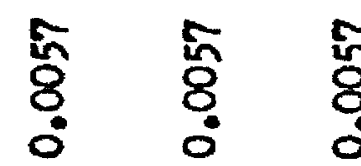

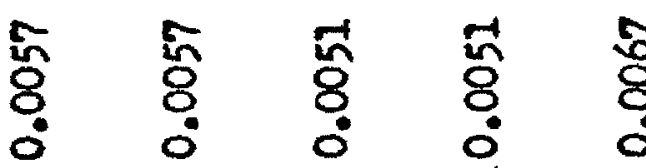

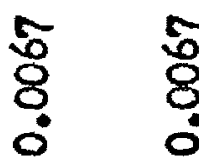

:

20

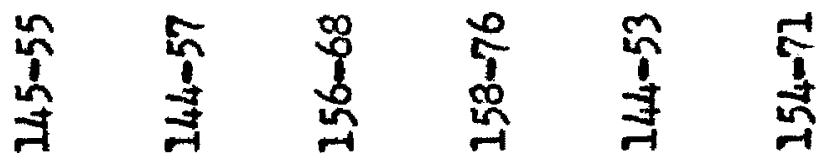

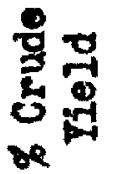

量必

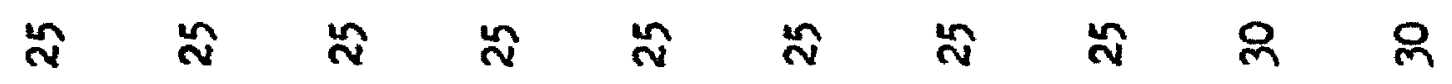

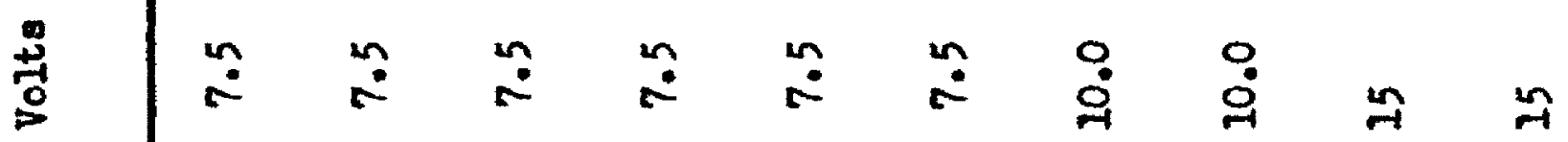

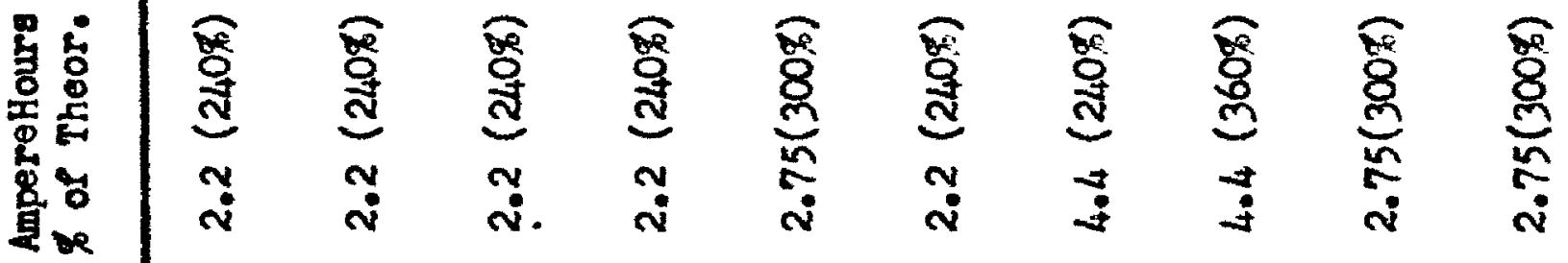

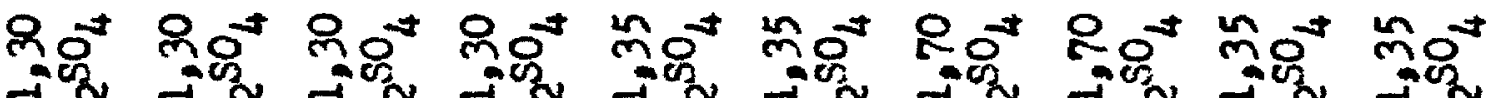

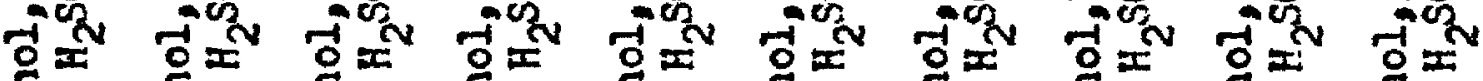

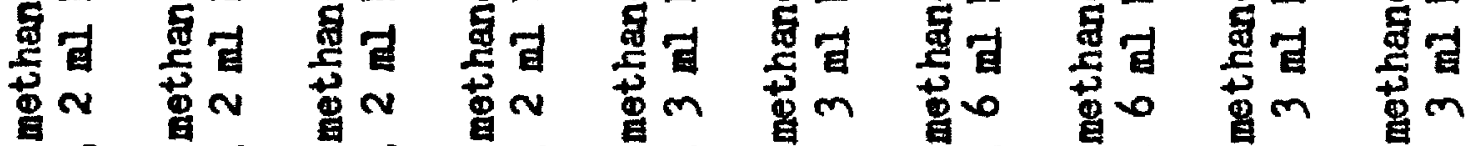
目完

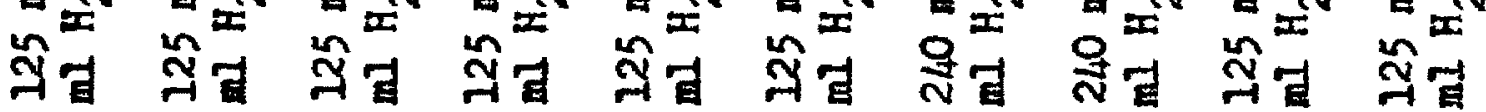

$\begin{array}{lllllllll}8 & 8 & 8 & 8 & 8 & 8 & 8 & 8 & 8\end{array}$

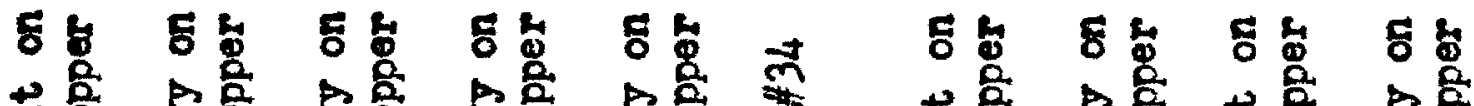
点亭

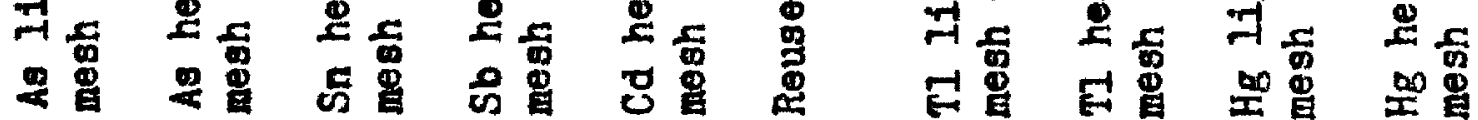
$\stackrel{0}{2}$
魯
\&
i
$\dot{\bar{m}} \dot{\mathrm{g}}$
$\dot{m} \dot{m}$
$\dot{m} \dot{m}$
$\dot{\hat{n}}$ 


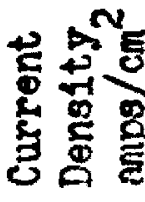

8

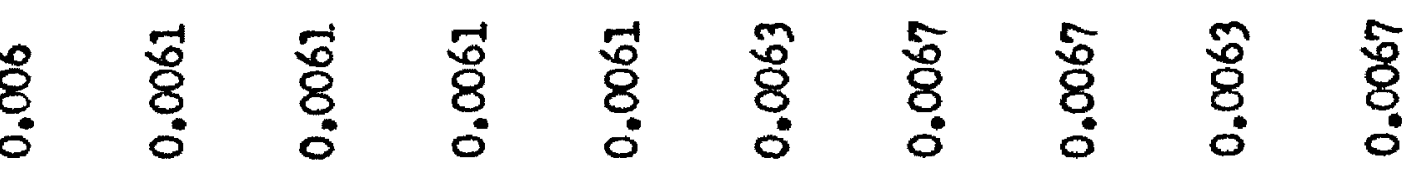

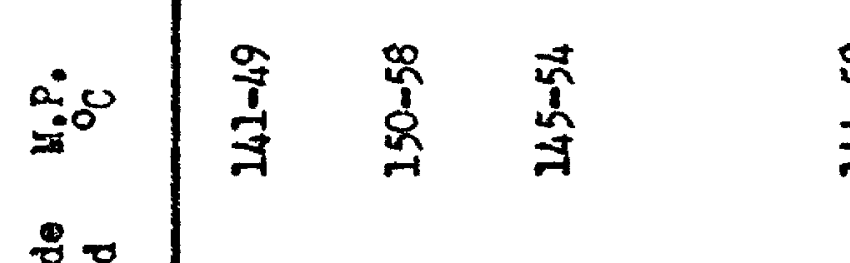

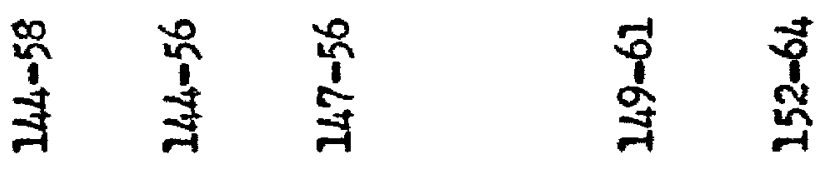

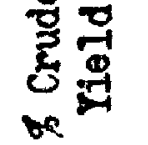

융 융요

$\begin{array}{lllll}0 & 0 & 0 & 0 & 0 \\ 0 & 0 & 0 & 0 & 0\end{array}$

통

ฉ

$\approx \approx$

※

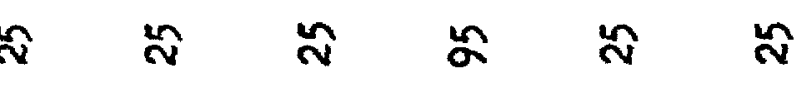

离

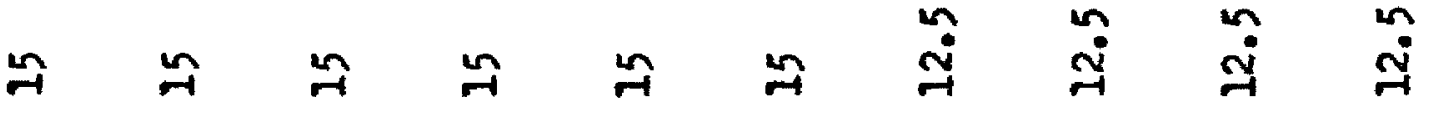

总害

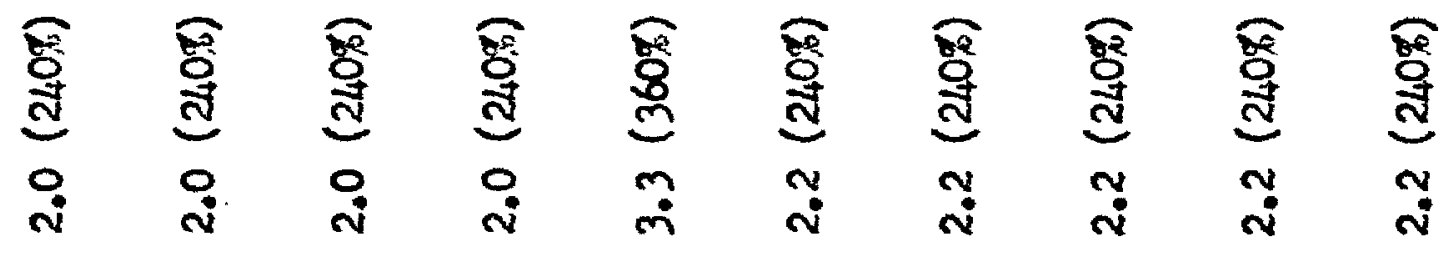

m̂t

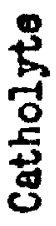

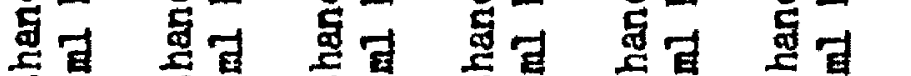

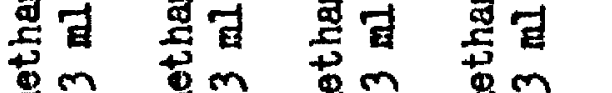

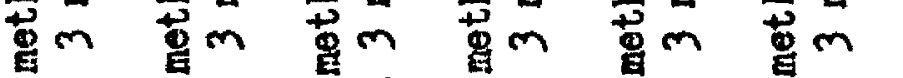

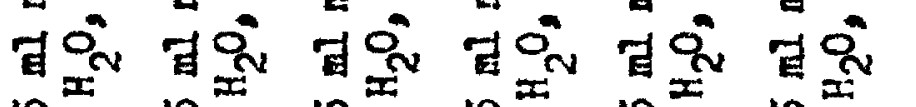

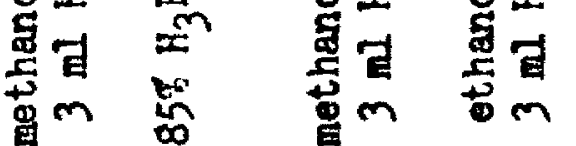

今ั

旁

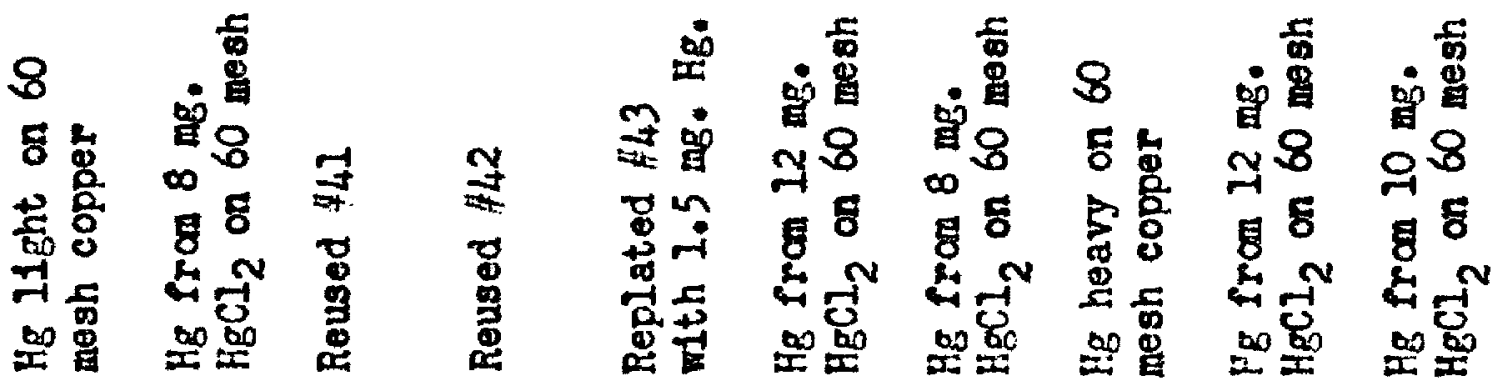

㔄 


\begin{tabular}{|c|c|c|c|c|c|c|c|c|}
\hline Run No. & Cathode & Catholgte & $\begin{array}{l}\text { Ampere Hour } 8 \\
x \text { of Theor. }\end{array}$ & Volts & ${ }_{{ }^{\circ} \mathrm{C}}^{\operatorname{Temp}}$ & $\begin{array}{l}\text { \% Crude } \\
\text { Yield }\end{array}$ & $\mathbf{u}_{{ }_{0}} P_{\bullet}$ & $\begin{array}{l}\text { Current } \\
\text { Densfty, } \\
\text { amps/cm }\end{array}$ \\
\hline 50. & $\begin{array}{l}\mathrm{Hg}-\mathrm{N1} \text { on } 60 \text { mesh } \\
\text { copper }(10 \mathrm{Hg})\end{array}$ & $\begin{array}{l}135 \mathrm{ml} \text { methanol,35 } \\
\text { ml } \mathrm{H}_{2} \mathrm{O}, 3 \mathrm{ml} \mathrm{H}_{2} \mathrm{SO}_{4}\end{array}$ & $2.2(240 \%)$ & 12.5 & 25 & 36.0 & $140-54$ & 0.0067 \\
\hline 52. & $\begin{array}{l}\mathrm{Hg}-\mathrm{N1} \text { on } 60 \mathrm{mesh} \\
\text { copper }(20 \mathrm{mg} . \mathrm{Hg})\end{array}$ & $\begin{array}{l}135 \mathrm{ml} \text { methanol,35 } \\
\text { ml } \mathrm{H}_{2} \mathrm{O}, 3 \mathrm{ml} \mathrm{H}_{2} \mathrm{SO}_{4}\end{array}$ & $2.2(2408)$ & 12.5 & 25 & 38.0 & $146-58$ & 0.0067 \\
\hline 52. & $\begin{array}{l}\mathrm{Hg}-\mathrm{Ag} \text { on } 60 \text { mesh } \\
\text { copper }(50 \mathrm{mg} \cdot \mathrm{Hg})\end{array}$ & $\begin{array}{l}135 \mathrm{ml} \text { methanol, } 35 \\
\text { ml } \mathrm{H}_{2} \mathrm{O}, 3 \mathrm{ml} \mathrm{H}_{2} \mathrm{SO}_{4}\end{array}$ & $2.2(240 \%)$ & 12.5 & 25 & $u_{4.0}$ & $262-74$ & 0.0067 \\
\hline 53. & $\begin{array}{l}\text { Hg heavy on } 60 \\
\text { mesh copper }\end{array}$ & $\begin{array}{l}85 \mathrm{ml} \text { methanol, } 40 \\
\mathrm{ml} \mathrm{H}_{2} \mathrm{O}, 15 \mathrm{ml} \mathrm{H}_{3} \mathrm{PO}_{4}\end{array}$ & $3.85(3108)$ & 17.5 & 15 & 31.5 & $140-80$ & 0.0067 \\
\hline 54. & Roused $\# 53$ & $\begin{array}{l}80 \mathrm{ml} \text { methanol, } 40 \\
\mathrm{ml} \mathrm{H}_{2} \mathrm{O}, 15 \mathrm{ml} \mathrm{HA} \\
6 \mathrm{ge}, \mathrm{NaAc}\end{array}$ & $2.75(220 \%)$ & 22.5 & 30 & 34.3 & $180-205$ & 0.0067 \\
\hline 55. & $\begin{array}{l}\text { Hg heavy on } 60 \\
\text { mesh copper }\end{array}$ & $\begin{array}{l}85 \mathrm{ml} \text { methanol, } 40 \\
\text { ml } \mathrm{H}_{2} \mathrm{O}, 3 \mathrm{ml} \mathrm{H}_{2} \mathrm{SO}_{4}\end{array}$ & $1.8(145 \%)$ & 10 & 20 & 22.9 & $154-193$ & 0.0073 \\
\hline 56. & $\begin{array}{l}\mathrm{Hg} \text { heavy on } 60 \\
\text { mesh copper }\end{array}$ & $\begin{array}{l}75 \mathrm{ml} \text { methanol, } 30 \\
\mathrm{ml} \mathrm{H} 0,15 \mathrm{ml} \mathrm{BAc} \\
6 \mathrm{~g} ., \mathrm{NaAc}\end{array}$ & $2.2(1778)$ & 15 & 25 & 21.4 & $165-204$ & 0.0067 \\
\hline 57. & $\begin{array}{l}\mathrm{Hg} \text { heary on } 60 \\
\text { mesh copper }\end{array}$ & $\begin{array}{l}85 \mathrm{ml} \mathrm{T} \text {-Butgl alc. } \\
30 \mathrm{ml} \mathrm{H} \mathrm{H}^{\mathrm{O}} 6 \mathrm{~g} \text { kiac } \\
15 \mathrm{ml} \mathrm{HAc}\end{array}$ & $1.8(1400)$ & 30 & 35 & 12.8 & $150-64$ & 0.0055 \\
\hline 58. & $\begin{array}{l}\text { Hg heavy on } 60 \\
\text { mesh copper }\end{array}$ & $\begin{array}{l}35 \mathrm{ml} \mathrm{T} \text {-But yl alc. } \\
45 \mathrm{ml} \text { methanol, } 18 \\
\mathrm{ml} \mathrm{KAc}, 6 \mathrm{~g} \text {. NaAc } \\
40 \mathrm{ml} \mathrm{H}_{2} \mathrm{O}\end{array}$ & $2.0(160 \%)$ & 35 & 30 & 33.0 & $150-84$ & 0.036 \\
\hline
\end{tabular}




\begin{tabular}{|c|c|c|c|c|c|c|c|c|}
\hline Run No. & Cathode & Catholyte & $\begin{array}{l}\text { Ampere Hour: } \\
\text { \$f of Theor. }\end{array}$ & Volt: & $\begin{array}{l}\text { Temp } \\
{ }^{\circ} \mathrm{C}\end{array}$ & $\begin{array}{l}\text { \% Crude } \\
\text { Tield }\end{array}$ & $M_{0} P_{C}$ & $\begin{array}{l}\text { Current } \\
\text { Deno1ty, } \\
\text { amps/cre }\end{array}$ \\
\hline 59. & $\begin{array}{l}\text { Hg heavy on } 60 \\
\text { mesh copper }\end{array}$ & $\begin{array}{l}160 \mathrm{ml} \text { methanol,60 } \\
\text { ml } \mathrm{H}_{2} \mathrm{O}, 6 \mathrm{ml} \mathrm{H}_{2} \mathrm{SO}_{4}\end{array}$ & $2.2(123 x)$ & 10 & 25 & 48.0 & $172-90$ & 0.0067 \\
\hline 60. & $\begin{array}{l}\text { Hi l1ght on } 60 \\
\text { mesh copper } \\
\text { (12 mg. Hg.) }\end{array}$ & $\begin{array}{l}160 \mathrm{ml} \text { methanol,60 } \\
\text { ml } \mathrm{H}_{2} \mathrm{O}, 6 \mathrm{ml} \mathrm{H} \mathrm{SO}_{4}\end{array}$ & $2.2(123 \not)$ & 10 & 25 & 25.0 & $161-200$ & 0.0058 \\
\hline 61. & $\begin{array}{l}\text { Hg heavy on } 40 \\
\text { mesh copper }\end{array}$ & $\begin{array}{l}170 \mathrm{ml} \text { methanol, } 60 \\
\mathrm{ml} \mathrm{H}_{2} \mathrm{O}, 6 \text { ml } \mathrm{H}_{2} \mathrm{SO}_{4}\end{array}$ & $4.0(227 x)$ & 10 & 25 & 38.0 & $150-87$ & 0.0060 \\
\hline 62. & $\begin{array}{l}\mathrm{Hg} \text { heavy on } 20 \\
\text { mesh copper }\end{array}$ & $\begin{array}{l}170 \mathrm{ml} \text { methanol,60 } \\
\mathrm{ml} \mathrm{H}_{2} \mathrm{O}, 6 \mathrm{ml} \mathrm{H}_{2} \mathrm{SO}_{4}\end{array}$ & $4.0(2278)$ & 10 & 25 & 0 & & 0.0077 \\
\hline 63. & $\begin{array}{l}\mathrm{Hg} \text { heavy on } 40 \\
\text { mesh copper }\end{array}$ & $\begin{array}{l}160 \mathrm{ml} \text { methanol, } 60 \\
\mathrm{ml} \mathrm{H}_{2} \mathrm{O}, 6 \mathrm{ml} \mathrm{H}_{2} \mathrm{SO}_{4}\end{array}$ & $4.0(2278)$ & 10 & 25 & 44.0 & $151-74$ & 0.0060 \\
\hline 64. & $\begin{array}{l}\text { Hg heavy on } 40 \\
\text { mesh copper }\end{array}$ & $\begin{array}{l}160 \mathrm{ml} \text { methanol, } 60 \\
\mathrm{ml} \mathrm{H}_{2} \mathrm{O}, 6 \mathrm{ml} \mathrm{H}_{2} \mathrm{SO}_{4}\end{array}$ & $4.0(227 \%)$ & 10 & 25 & 49.0 & $14 h-79$ & 0.0060 \\
\hline 65. & $\begin{array}{l}\text { Hg heavy on } 60 \\
\text { mesh copper }\end{array}$ & $2 \mathrm{~N} \mathrm{NaOH}$ & $2.1(1008)$ & 12.5 & 30 & 35.0 & $173-79$ & 0.0604 \\
\hline 66. & $\mathrm{Hg}$ pool & $2 \mathrm{~N} \mathrm{NaOH}$ & $2.1(153 \not 6)$ & 12.5 & 30 & \multicolumn{2}{|c|}{$\begin{array}{l}\text { Acetylated \& } \\
\text { Dehydrated }\end{array}$} & 0.1783 \\
\hline 67. & $\mathrm{Hg}$ pool & $2 \mathrm{NaOH}$ & $2.1(1399)$ & 12.5 & 30 & \multicolumn{2}{|c|}{$\begin{array}{l}\text { Schotten- } \\
\text { Baumann }\end{array}$} & 0.1783 \\
\hline 68. & $\begin{array}{l}\mathrm{Hg} \text { heavy on } 60 \\
\text { mesh copper }\end{array}$ & $2 \mathrm{~N} \mathrm{NaOH}$ & $2.1(2009)$ & 12.5 & 30 & 44.0 & $150-88$ & 0.0362 \\
\hline
\end{tabular}




\begin{tabular}{|c|c|c|c|c|c|c|c|c|}
\hline Run No. & Cathode & Catholyto & $\begin{array}{l}\text { AmpereHours } \\
\text { \& of Theor. }\end{array}$ & Volts & ${ }_{{ }_{0} \mathrm{C}}^{\text {Temp }}$ & $\begin{array}{l}\text { Crude } \\
\text { Tield }\end{array}$ & $\mathrm{H}_{{ }_{0}} P_{0}$ & $\begin{array}{l}\text { Current } \\
\text { Density } \\
\text { amps } / \mathrm{cm}^{2}\end{array}$ \\
\hline 69. & $\mathrm{Hg}$ pool & $2 \mathrm{~N} \mathrm{NaOH}$ & $2.1(230 \%)$ & 12.5 & 30 & 98.0 & $150-74$ & 0.1783 \\
\hline 70. & $\begin{array}{l}\text { Hg heavy on } 60 \\
\text { mesh copper }\end{array}$ & $2 \times \mathrm{NaOH}$ & $1.8(1008)$ & & 25 & 50.0 & $150-60$ & 0.00093 \\
\hline 71. & $\begin{array}{l}\mathrm{Hg} \text { heavy on } 60 \\
\text { mesh copper }\end{array}$ & $2 \mathrm{NaOH}$ & $2.1(120 \%)$ & 12.5 & 25 & 74.0 & $142-159$ & 0.106 \\
\hline 72. & $\begin{array}{l}\mathrm{Hg} \text { heavy on } 60 \\
\text { mesh copper }\end{array}$ & $2 \mathrm{NaOH}$ & $2.65(150 \%)$ & 12.5 & 25 & 12.0 & $186-200$ & 0.106 \\
\hline 73. & $\mathrm{Hg}-\mathrm{Na}$ pool & $2 \mathrm{~N} \mathrm{NaOH}$ & $2.1(127 \%)$ & 12.5 & 25 & 68.0 & $153-63$ & 0.1684 \\
\hline 74. & $\mathrm{Hg}-\mathrm{Na}$ pool & $2 \mathrm{~N} \mathrm{MaOH}$ & $2.5(1408)$ & 12.5 & 25 & 7.0 & $172-79$ & 0.1684 \\
\hline 75. & $\begin{array}{l}\mathrm{Hg} \text { heavy on } 60 \\
\text { mesh copper }\end{array}$ & $2 \mathrm{~N} \mathrm{NaOH}$ & $2.5(1408)$ & 7.5 & 30 & 63.0 & $150-65$ & 0.0076 \\
\hline 76. & $\begin{array}{l}\text { Hg heavy on } 60 \\
\text { mesh copper }\end{array}$ & $2 \mathrm{~N} \mathrm{NaOH}$ & $2.5(140 \%)$ & 7.5 & 30 & 87.0 & $150-65$ & 0.0076 \\
\hline 77. & $\begin{array}{l}\text { Hg heavy on } 60 \\
\text { mesh coppor }\end{array}$ & $2 \mathrm{~N} \mathrm{NaOH}$ & $4.2(310 x)$ & 7.5 & 15 & 90.0 & $150-165$ & 0.2415 \\
\hline 78. & $\begin{array}{l}\text { Hg heavy on } 60 \\
\text { mesh copper }\end{array}$ & $5 \mathrm{~N} \mathrm{NaOH}$ & $2.8(225)$ & 7.5 & 15 & 17.2 & $170-85$ & 0.0073 \\
\hline 79. & $\begin{array}{l}\mathrm{Hg} \text { heavy on } 60 \\
\text { mesh copper }\end{array}$ & $30 \% \mathrm{NaOH}$ & $2.75(220 x)$ & 10 & 30 & 26.0 & $146-71$ & 0.0067 \\
\hline 80. & $\begin{array}{l}\text { Hg heavy on } 60 \\
\text { mesh copper }\end{array}$ & $2 \mathrm{~N} \mathrm{NaOH}$ & $1.65(100 \%)$ & 7.5 & 20 & 95.0 & $155-70$ & 0.007 \\
\hline
\end{tabular}




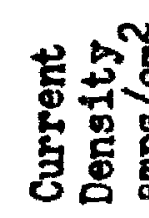

F

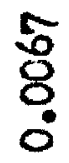

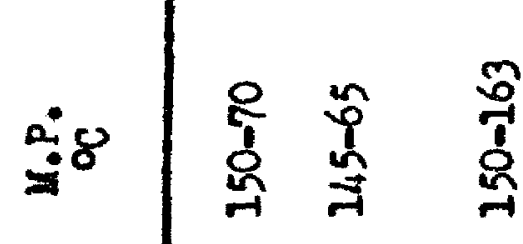

$\frac{8}{8}$

总兽

$\begin{array}{lll}0 & 0 & 0 \\ \text { ô } & 8 & 0\end{array}$

\%

角次

ลิ ผี

ผ

w

咅

n

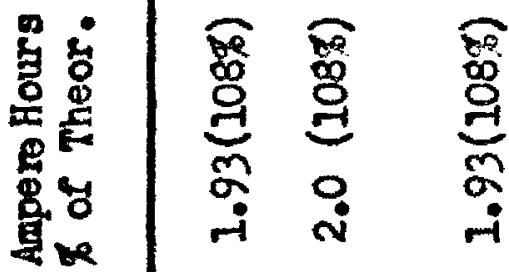

in

m

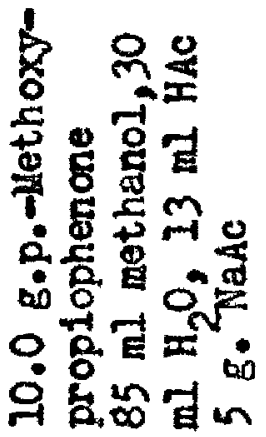

88

8

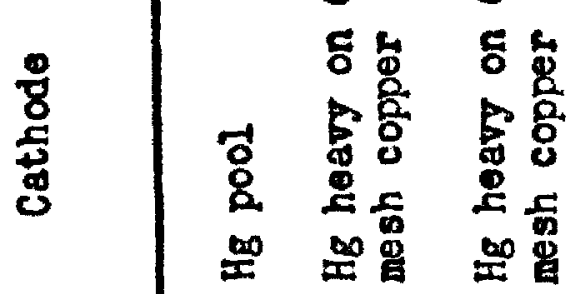

몽

察

g

\&

옵

울

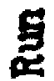

$\frac{1}{\infty}$

$\frac{1}{\infty}$

$-1$ 
Vith lead pleted cathodes, no definite minimun of lead concentration was established so that just light and heavy platings were used; a light plating being one where copper was visible. In order to obtain uniforaly 11ght plated electrodes, heavily flated oneg vere first prepared and then alpped in a solution of 3-5\% copper sulfate. These dipped cathodes were reactive and fair reductions in acid nedium were obtained. Heavily coated lead gave poorer results in acld medium and lead cathodes of either type gave very low current officlencies in alkaline medium. Results with cadmilum were cuite similar to those of lead, whlle smaller ylelds were obtained with blsmuth and thallium. The cathodes plated with arsenic, antimony, and tin did not produce the desired reation. Eodium.- Next to the nature of the aathode, the fector most affecting the course of the reduction was the composition of the catholyte. The acid medium consisted of methanol and water as the solvent for the ketone and sulfuric acid as the electrolyte. The amount of suliuric acid nocessery was kept to a near minimum, but witien only $1 \mathrm{ml}$. was used there was a sharp aroo in current efficlency. Larger amounts than $5 \mathrm{ml}$. of sulfuric acia were not used as, at this point, a doorease in yield of plnacil was obtained although current efficioncy remsined hich. Thus $3 \mathrm{ml}$. of concentrated sulfuric acld per $5 \mathrm{~g}$. ketone vas found to give the bost results. Simllar reactions asing 
phosphorlc acld in place of the sulfuric acld gave low current efficiency, excopt when using heavily plated mercury cathodes. The use of syrupy phosphoric acld as tho electrolyte and solvent was hindered by excesilve foaning due to the low current effleiency and startine meterial vas recovered. Sthanol and water was also used as the solvent, but without increasing the yiela.

For slkaline mediun, $2 \mathrm{~N}$ sodium hydroxide was used as solvent and electrolyte. As the concentration of the sodiun hydroxide was increased, the pinacol precipitated out, however, this was a disadiantago as the cathode became coated with product and thus roduction vas hindered. This medium proved the most userul as it wets easy to frepare, economical, and the product easily recovered and purified.

In addition to strongly ac $\pi r^{\circ}$ alkaline media, buffered solutions, with a pll of 5.6-." of acetic acid and sodium acetate were also use: . Hese were characterized by only fair current efficien: aith mercury cathodes and very low current fficiency with lond olectrodes.

Temperature.- Lowering the temperature belo: 200 had no noticeable effect on the course of the resuction. sbove $35-40^{\circ}$ the yields drolped off in acid mediua. No study was made of the effects of tenperature on yleld in alkaline medium, but all runs wero made between $20-30^{\circ}$. 
Current density.- High current density hud little effect in either acid or alkaline medium on the total yield or proportion of the two isomers formed, however, if it was too low, no reduction occured. Current density vas regulated both by the amperage passed tirongh the cell and the surface area of the cathode. By reducing the area of the cathode, a high current density could be obtained without high amperage so that overheating of tho cell was avoided.

Purification of pinacol.- Aftor separating tho non-crystaline by-products and unreacted starting naterial, the crude product melted over a range of $10-15^{\circ}$ from about $150-165^{\circ}$, with each run having a slightly different melting point. Since two isomeric pinacols can be formed from this reduction, it ssemed likely that this product was a mixture. Attenpts to effect a senaration by recrytallizing from acetic ecld were unsuccessful. ith each racrystallization, there pas a loss of $50: 3$ in material without a significant rise in melting point. since it seemed possible that a pinacolone ranrangenent occured during the hesting with aotic acid, acetone and acetono-1so-propyl ether wore tried as solvents. skain there was extensive loss of material without arpreciable improvement in the celtine roint. Chromatogreyile methods were also employed in attempts to effect a separation. Silloa fel was uged as the adsorbent, with ether-acetono as eluant. Again, no effective separation could be aado 
although there was a olight lmprovernent in the melting point. During runs using $20 \%$ sodium hydroxide solution as electrolyte, it had been noted that the nigacol formed precipitated out. This insolubility in strong alkall was therefore tried as a basis for making a separation. If the crude product was dissolved in dilute sodiun hydroxide and then enough solid sodium hydroxide added to make a $20 \%$ solution, no solld seperated on cooling. However, it was found that cold 20; sodium hydroxide had a selective solvent action ror one (1somer B) and that is the crude mixtures were extracted three times with 20,0 alkali, a fairly clean soparation could be made, with little loss of product.

Isomer $B$ separated on acidifying the elkalino solution to give a product melting at about $175-135^{\circ}$. The crude materlal gave approximately erual amounts of the two 1somers. Purification of these isomers was difficult due to their tendency to occlude solvents and because they had little tendency to separate from each other on recrystallization. The purest sample of lsomer is obtained melted at $204-208^{\circ} \cdot\left(204-206^{\circ} 11 t .\right)^{6}$

Proof of structure.- Two isomers of this pinacol have been reorted in the literature.7 The wego configuration has been assigned to one melting at 204-206., although there is no direct proof that this is the proper configuration. This isoner is belleved to be identical with isomer A, but again this could not be proven without 
a direct comparison such as a mized melting point. The di 130mer has been reported to melt at $96-97^{\circ}$, but no such compound was lsoluted from any of these reaction mixtures, and the coapound separated from the cold altali, 1somer $B$, wo ld apparentiy have a melting point about as hi has isower if it could be obtalned in a purer state. "hen compounds and $B$ were mixed, the melting point ves depressed to 162-199\%. Proor that compouns i and $B$ were isomeric wes obtained by heuting esch in alcoholic solution with p-toluenesulfonic acid. Under those conditions, a pinacolone rearrangement occured to form compound (IV), 3. 3 b1s-(p-hgaroxypheny $)$-4-hexanone. The reaction inf $x-$ ture was an ofl, but treatment with benzogl chloride croduced a dibenzoate (VII) neiting et $173.5-175^{\circ}$ from compound $A$ and $174-175.5^{\circ}$ from compound $B\left(170^{\circ} 11 t_{0}\right) .^{8}$ Fo depression was observed from a mixed nolting point. is diacetate of compound $A(V I)$ was also obtalned, m.p. $84.5-86^{\circ}\left(91^{\circ} 11 t.\right) .9$

Dehydration of the isomeric mixture of $A$ and $B$ ond purified $A$ using the nethod of Dodas, 10 refluxing in cetic anhydride-acetyl chloride solution, both produced dionostrol diacetate (III) in moderate yield, m.r. 117-119 $\left(119-120^{\circ}\right.$ 1it.). 11 Fydrolysis of the diacetate proanced dienestrol (V) u.p. $230-231.5^{\circ}$ vac. $\left(227-228^{\circ} 13 t_{0}\right) .12$ jince there was no difference in yields obtilned on dehydration of the mixture of 1 somera and yurified is, it may 
be assumed that both isomers dehydrete with about equal ease although no attempt was made to dehydrate purified Lsomer B.

SULAARY

Plated copper gauze cathodes have been found to be highly effective in bringing about the pinacol reduction of the ketone, p-hydroxypropiophenone. Nercury was found to be the most effective plating materisl. Both di and meso isomers were produced in abont enual cuantities under a variety of conditions, with reduction in alkaline medium giving the best results. Results indicate that further work could be done profitably with these electrodes on other compounds which are subject to this type or reduction. 


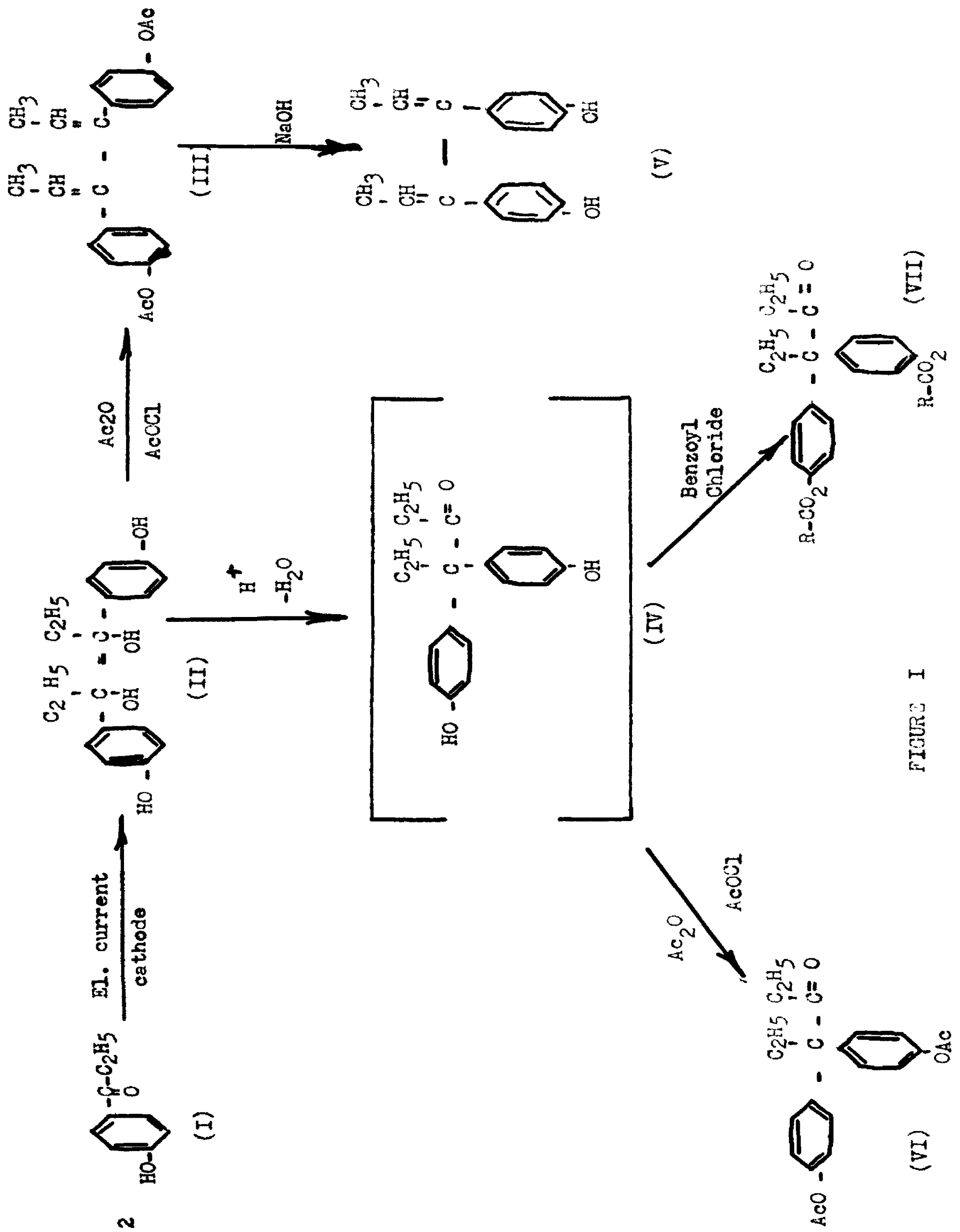




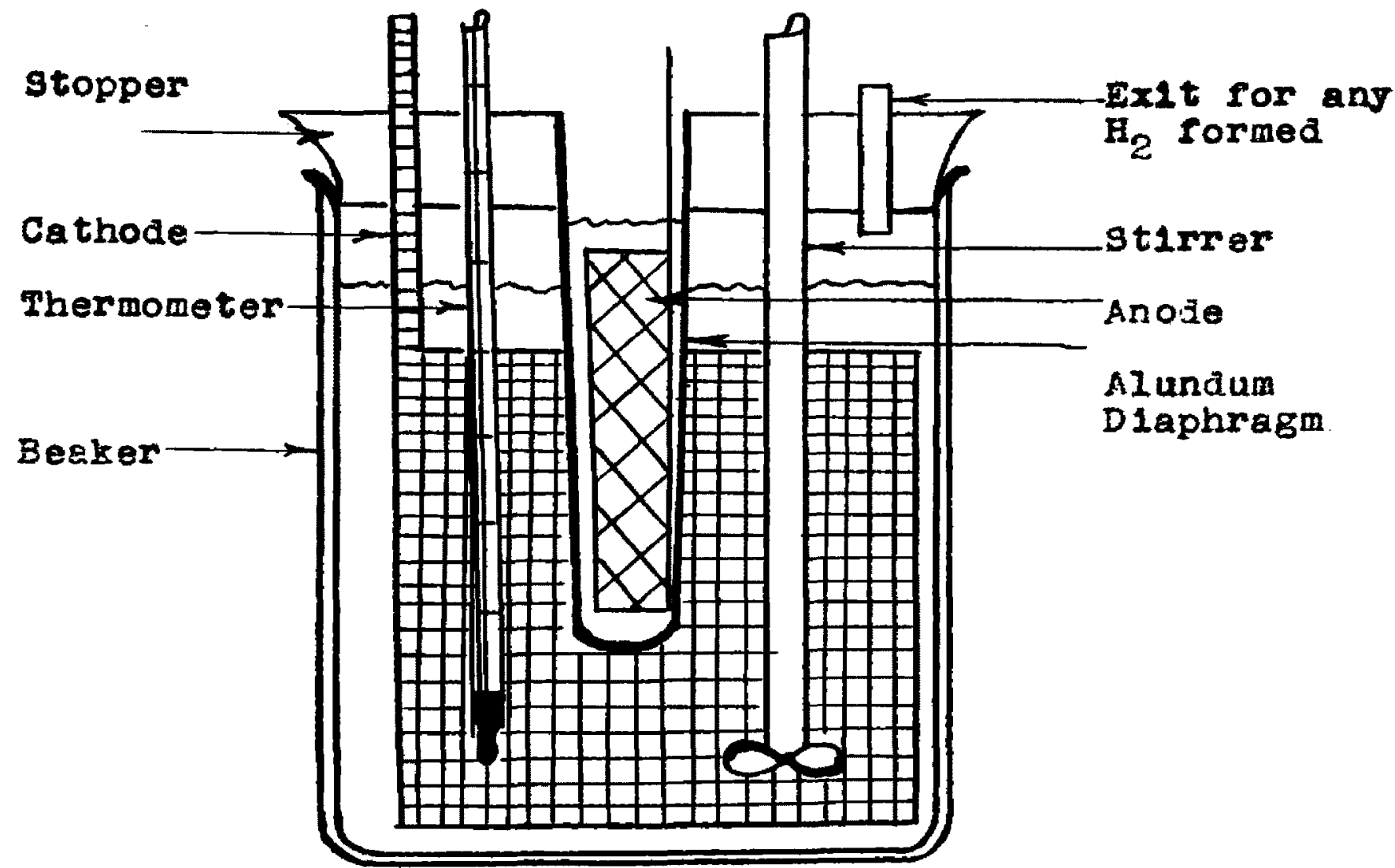

FIGURE II

APPAKATUS.

Electrolytio cell.- The electrolytio cell con sieted of the following: a $300 \mathrm{ml}$. beaker used as the cathode compartment, No. 25 rubber otopper, anode, cathode, meohanlcal etirrer, alundum diaphragm, amp. meter, volt meter, thermometer, and a source of variable direct current. Thie wa assembled according to the diagram. 
Cathode.- As the otructural material for the cathode copper gauze of 20,40 , and 60 mesh per 1 nch was used. In calculating the surface area of these cathodes, each wire had a certain amount of stretch when removed from the mesh. which was due to the overlapplng of one wire over another to form the mesh. Thus, a factor was used for each to determine the actual length from the apparent or measured length. The factora were 1.1 for 20 mesh, 1.19 for 40 mesh, 1.25 for 60 mesh. The diameter of the 20 mesh wire was 0.0155 inches, the 40 mesh was 0.009 inches, and the 60 mesh was 0.007 inches.

The cathode of the desired mesh was shaped a a cylinder, with a diameter allghtly less than the inside of the beaker and sewed together with fine copper w1re. It was cleaned by dipping in a dilute solution of nitric ac1d, washed with water, and the metal of cholce plated upon 1t. Lead was plated from a 5-10, solution of lead fluoborate at 1.4-3.0 amp. unt1l the desired coating was obtalned. For uniformity of 11ghtly plated cathodes, they were all heav1ly plated then dipped in a 5\% solution of copper alfate. Llghtly mercury plated cathodes were prepared by dipping the ac1d cleaned electrode in the desired solution of mercurio chloride. Solutions contalning $2,8,12,20$ and $50 \mathrm{mg}$. of mercurlc chloride were used. Heavily mercury plated cathodes were obtalned from solutions containing 3 . of mercuric chloride. B1smuth 
cathodes were electroplated from solutions of bismuth n1trato and also from $5 \%$ solutions of bismuth subcarbonate in $33 ;$ perchloric acid. Nickel amalgan cathodes were prepared by first electroplating nlckel heavily on the copper gauze at 2.2 amp. Pron a $10 \%$ acrueous solution or nickelous anmonium sulfate and $20 \mathrm{ml}$. of ammonium hydroxide. This was then dipped in a solution containing 50 mg. of mercuric chloride to obtain the amalgamated cathode. is silver amalgam vas similarily prepared. The silver vas flrst plated from a solution of silvor cyanide which was prepared by dissolvins silver nitrate in water, then neutralizing with potassium hydroxide, and then adding an excess of potasilum cyanide.

Arsenic was electroplated from a solution of $3 \mathrm{~g}$. sodium arsenate dissolved with a miniccum amount of hyârochloric acid and then diluted to $400 \mathrm{ml}$. It was difficult to get a unform plating of arsenic. Tin was electroplated from a solution containing stannous oxide, while antimony was plated from a solution, antiaony oxide, dissolved in hydrochloric acid then alluted to $400 \mathrm{ml}$. ill were electroplated by pasing a current of 2.2 ary.

Cadmlum vas electroplated at 0.7 arm. from $\approx 5^{\circ}$ solution of cadmiun oxide dissolved in ferchloric acid and then diluted with water. In nreparing the solution for thallium plating, $2 \mathrm{~g}$. of thallio oxide wes alssolved in a minimum amount of hydrochloric ac1d, then renrecigjt ton 
by neutralizing with sodium hydroxide. This precipitate wa then centriruged, washed, and dissolved in a finfmum amount of perchlorio acld. This solution wes diluted to $400 \mathrm{ml}$. and the thallium electroplated at 2.2 amp. Some of these electrodes wore also dipped in a $3 ;$ solution of copper sulfate.

Anode.- Platinum was used as the anode in all reductions and electroplating with the exception of those using lead cathodes in sulfurto or phosphoric acid solution.

Diaphragas.- Alundun extraction thimbles $20 \times 80$ mo. and $34 \times 100 \mathrm{~mm}$. of dense porosity were used as the diaphragm and also served as the anode compartment. Tho method used in decreasing the porosity of the diaphrafm for use in aold medium, 13 was to allow the thimble to soak in a $20 \%$ solution of water glass for one hour, then remove it and place it in a $20 \%$ solution of sulfuric acid for one hour. At the end of this time, it was removed and allowed to dry for three hours, then stored in a $20 \%$ solution of sulfuric acld. Th1s procedure was necessary only in the case of the larger thimbles.

Reduction

ficld mediun.- The catholyte conststed of $10 \mathrm{~g}$. of p-hyaroxypropiophonone aissolved in $200 \mathrm{ml}$. of mothanol, $70 \mathrm{ml}$. of water, and $6 \mathrm{ml}$. of coacentrated sulfuric acid. The anolyte was $40 \mathrm{ml}$. of methanol, 
$24 \mathrm{ml}$. Of water, and $1 \mathrm{ml}$ of concentrated sulfuric acia. The cathode and anode were as previously described. The reduction was allowed to proceed at $25^{\circ} \mathrm{using}$ a water bath to control the temperature, and stirred vigorously until current efficiency appeared to have dropped to zero. The inltial current was $2.6 \mathrm{emp}$. at a potential of $B$ volts. Upon completion of the reduction, the catholyte was neutrallzed with ammonium hydroxide, flltered and the methanol romoved in vacuo. The resulting brown gummy material was extracted with ether, the ether solution cried with argnesium sulfate, filtered and then the ether evaporated in vacus. The residue was crystallized from a minimum amount of acetic acid with care beling taken not to heat over 35-40 , and the crystalline material separated by suction filtration. After drying, a yield of 44 with a m.p. 147-59 was obtalned. This product was then recrystallized from acetic acid to obtain a m.p. 160-69\%, hoirever, there was a 50\% loss of product. The purfelcation of this crude product is best obtained by following tro procedure described below, usin, 20, sodiun hydroxide. ilkaline medium.- The catholyte consieted of 10 . of p-hyaroxypropiophenone dissolved in $135 \mathrm{nl}$. of 2 If sodiun hydroxido. Tho cathode was heavily plated mercury with platinum anode. The reduction was corried out by passing a current of 2.2 erap. at a potenti.1 of 7.5 volts for 47 minutes. The mixture was vigorously 
stirred and the temperature of $25^{\circ}$ maintained with the ald of water bath. At the completion of the reduction, the catholyte was neutrallzed with acetic acid, heated unt1l the gummy precipltate solidified, then troken up and $10 \mathrm{~g}$. of sodium chlorido added. The precipitate was then filtered, washed with hot water, and dried. A orude gleld of $9.9 \mathrm{~g} .(99 \%)$ of glycol, m.p. 148-59\%, was obtined. This product was then extracted with cold $20 ;$ sodiun hydroxide and after acldifying the sodium hydroxide insoluble material with aoetic acid, a yield of $4.6 \mathrm{~g}$. m.p. 171-79 ${ }^{\circ}$ was obtained. Upon extracting twice more, In a similar manner, a final yield of $3.1 \mathrm{~g}$. of 1 somer A, m.p. $198-204^{\circ}$ was obtalned. The alkaline flitrates were combined, neutralized with acetic acid, and cooled. After filtering, washing, and drying $2.7 \mathrm{~g}$. of 1somer B, m.p. $161-73^{\circ}$ was obtained. This was dissolved in reagent acetone, in which it was very soluble, and bolled down whlle lsopropyl ether was added. This process was continued unt1l all the acetone was evaporated with much care beling taken to aroid bumping. The solvent vas decanted while hot and the residue washed with 1sopropyl ether. A recovery of $2.2 \mathrm{~g}$. with a m.p. 159-710 was obtalned. No further purification of this portion of the product was attempted.

Buffered medium.- The catholyte consisted of 70 ml. of methanol, $30 \mathrm{ml}$. of water, $15 \mathrm{ml}$ acetic acid, $6 \mathrm{~g}$. 
sodium acetate, and 7 B. p-hydroxyroniophenone. The anolyte consisted of $4 \mathrm{ml}$. methanol, $4 \mathrm{ml}$. D-butyl alochol, $8 \mathrm{ml}$. of vater, $3 \mathrm{ml}$. acetio acla, und $1 \mathrm{~g}$. sodium acetate. A hesvily plated mercury cathode was usod. The reduction was carried out by passing e current of 2.2 anp. at a potential of 15 volts for one hour. is tomperature of $30^{\circ}$ was maintained with the aid of a vater bath and vigorous atirring. The pll at the start of the reaction was 5.6 and at the end 5.8 . At tho completion of the raduction, the catholyte sas perty neutralized with sodiun sydroxide and completed ith sodium blcarbonate, and the methanol exarorated in vacuo. This resulting product was extracted with ether, ariea with magnesiun sulfoto, and the ether evaporated in vacuo. Boiling tolueno was used for separating the crystalline and non-crystaliine roducts, however, due to the poor recovery of pruduct, the toluene was evaporated in vacuo and acetic acia used to recover the renainder as in the acid roductions. "yield of 1.4 G. M.F. $165-177^{\circ}$ \%as obtained.

\section{4 bis (p-methoxphenyl) 3, 4 hexanediol.- The}

catholyte consisted of 10 B. p-methoxypropiophenone, $85 \mathrm{ml}$. of methanol, 13 ril. of acetic acid, $5 \mathrm{~g}$. sodiun acetate, and $30 \mathrm{ml}$. of water. The anolyte was $3 \mathrm{ml}$ of acetic acia, $1 \mathrm{~g}$. of sodium acetate, $10 \mathrm{ml}$. of water, and $3 \mathrm{ml}$. of - milgl alcohol. The reduction was carried out by passing a current of 2.2 amp. at a potential of 15 volts for 93 minutes. The temperature of $25^{\circ}$ yas nalatained fith the aid of vater. 
bath. Due to the precipitation of the rinacol, the outhode beceme heavily coated after 30 minutes and interfered with the reduction by lowering current efficienoy. ist the completion of the reaction, the oatbolyte was neutralized with sodiun hydroxide, the nethanol evaporated In vacuo, and $100 \mathrm{ml}$. of wator added. It wis then heated to near bolling, $10 \mathrm{~g}$. sodiur chloride addod, and upon cooling a precipltate forwed which vas filtered, washed vith weter, and dried. A yield of $2.6 \mathrm{~g}$. wh th a.p. 179-1900 was obtainod, bovever, no further purification was attemoted.

Purificetion of the product.-

As a test for starting naterial, 2, 4 ainitropbenglhydrazine was used and when found prosent, it was removed by forming the water soluble semicarbezone. This reruired 4. g. of semicarbazide hydrochloride dissolved in a minimua amount of water and $6 \mathrm{G}$. sodiua acetate dissolved in a minimum acount of methanol. Those solutions were poured together, filtered, and added to $B E$. of the crude pinacol In a $100 \mathrm{ml}$. round bottom flask. This was refluxed for 75 minutes and then poured into $300 \mathrm{ml}$. of wam vater. Upon cooling, a rocipltate forned which was thon extricted using cold 20; sodlum hydroxide as the solvent. Tho insoluble material was filtcred of usin: a sinterod gloss funnel, and the vashing with $20 \%$ sodiun hydroxide reveted twice more. The insoluble product was dissolved in ater, the solution acidified iith acetic acid, and the solid 
which sepereted, flltered, washed with water, and dried to obtain $2.7 \mathrm{~g}$. and a m.p. 195-204\%. The alkaline filtrates were combined and neutralized with acetic acid, cooled and the resulting precipltate, 1somer $B$, filtered, washed with water and dried. A yleld of $2.1 \mathrm{~g}$. with a melting point of $180-205^{\circ}$ was obtained; but no further purification was attempted. Isomer a was recrystallized Irom reagent acetone with consiajerable loss, howerer, this was recovered by bolling off the acetone and adding 1aopropyl ether. The recrystallized isower A melted at $190-200^{\circ}$.

Ereparation of derivatives.-

Dienestrol diacetate.- The pinacol vas acetylated and dehydrated according to the method of Eodda.14 In a $100 \mathrm{ml}$. round bottom flask, $10 \mathrm{~g}$. of the pinacol was dissolved in $35 \mathrm{ml}$. of acetic anhydride and $4 \mathrm{ml}$. of acotyl chloride. After refluxing for three hours, the acetyl chloride and acotic anhydride were distilled of in vacuo. To the residue, $30-40 \mathrm{ml}$. of vater was added, neutralized with sodium bicarbonate, and wawed by decantation with water. The resultant product nas dissolved in $25 \mathrm{ml}$. of ethanol and then bolled down to $15 \mathrm{ml}$. from which the dienestrol diacetate precipitated. The crude product was recrystallized from ethanol to yield $3.6 \mathrm{~g}$. with a m.p. $117-119^{\circ}\left(119-120^{\circ} 11 t.\right)$.

Dlenestrol.- The dlacetate was hyarolyzod by refluxing with 7.5: sodium hydroxide for 14 hours to obtiln 
3, 4 bis (p-hydroxphenyl) 2, 4 keradine, (dienestrol) m.p. $215-224^{\circ}$ in a $78^{\circ}$ yiela. This was recrystal117ed from isopropyl ether to give a m.p. 223.5-226 $227-8$ 11t.). 16 with decomposition. ithen dried in vicho and tho caplliary seal ed a m.p. 230-231. $5^{\circ}$ without decomposition was obtained.

\section{2 bis (e-hydroxyphenyl) 4 hexanone, dibenzoate.-} Ono gram samples of isomer $A$ and $B$ were dissolved separateIy in $20 \mathrm{ml}$. of ethanol and to each 301 ution $0.3 \mathrm{~g}$. of p-toluene-sulfonic acid was added. After bolling for 20 minutes, these solutjons were poured into $200 \mathrm{ml}$. of warn water and allowed to cool. The olly substance which sepurated was extracted with ether, aried with nagnosium sulfate, and the ether evaporated in vacuo. The residue was dissolved in $10 \mathrm{ml}$. of potessium hydroxide and benzoyl chloride added dropwise with vigorous shaking. The dibenzoates so prepared were recrystallized from ethanol, to obta1n $1.1 \mathrm{~g} \cdot\left(65^{\circ}\right) \mathrm{a} . \mathrm{p} \cdot 171.5-174^{\circ}$, 1 somer A; $1.2 \mathrm{~g}$. $(70,0)$ M.P. $171-174^{\circ}$, Isomer B. The dibenzoate of $B$ was recrystallized from benzene to give a product melting at 174-175.5 $5^{\circ}\left(178^{\circ} 11 t.\right) .17$ tio depression of welting point was obteined by the mixed melting roint of the ketone dibenzoates obtained from isomer is and $B$.

3. 2 bis (2-hydroxybengl) L hexanone, diacetateOne gram of lsomer $i$ wis dissolved in $20 \mathrm{ml}$. of ethanol and $0.3 \mathrm{E}$. of p-toluenesulfonic acid adied. Neter boiline 
for 20 minutes, the solution vas poured into $230 \mathrm{ml}$. or wara water and cooled. Thia oliy resiaue vas extracted with ether, and the ether evaporated in vacus, then refluxed vith $15 \mathrm{ml}$. of acetic anhydride and $2 \mathrm{ml}$. acetyl chloride for 90 minutes. The acetyl chloride and acetic anhydride was distilled off in vacuo, 30 ml. of witer added, and neutralized with sodium bloarbonate. This was then washed with water by decantation and tho product recrystallized from ethanol to give approxinately a 75 ; yield with a m.p. $84.5-86^{\circ}\left(91^{\circ}\right.$ lit. $) .18$ 


\section{LITETATUE CITED}

12. Backer, H. J., Proc. Chem. Soc. 28,65 J. Chem. Soo. 101, 582-9.

b. Bradt and Andergon, Trans. Electrochos. Soo. Pp 75, 10 (preprint) 1339.

2. Robinson, F. A. and Resugzan, J.C. L., British Patent 523, 515 (Glaxo Laboratorles) Feb. 9, 1940.

3. Swann, S. Jr.. Electrolyt10 Reactions, p 143-207 Sechniaue of Organic Chemistry. vol. II Interscience Publishers, lew York.

4. Caspar1, Z. Phy 81k. Chem. Pp 30, 89 (2899).

5. Tafel, 2. Phye1k. Chem. Pp 50,641 (1905).

6. Dodds, E. C., Goldbery, I., Lawson, , and Roblnson R., Proc. Roy. Soc. (Londón) B 127, 140 (1939).

7. Hobday, G. I. and Short, W. F., J. Chom. Soc. 1943 Pp 609-12.

8.. Hobday, G. I. and Short, W. F., J. Chem. Soo. 1943 Pp 609-12.

9. Hessley, S. V., Kerschbaum, E., Kleedorfer, A., Prillinger, $F$, Zaj10, E. Monatsh. 73, $127-58(1940)$.

20. Dodds, E. C.. Goldbery, L., Lawson, H. and Robinson E.. proc. Róy Soc. (Lóndón) B 127-58 (1940).

11. Wessley, F. V., Kerschbeum, A., Kleedorfer, A.. Prililinger, F, Zaj1c, E. Monatsh, 73, 127-58 (1940).

12. Dodds, E. C., Goldbery, L., Lawson, , and Robinson R., Proc. Roy Soc., (London) B 127, 140 (1939). 
13. Swann, 3.. Jr.. Electrolyt10 React1ong, p 143-207 Technique of Organic Chenistry, Vol. II Interscience Publishers, New York.

14. Dodds, E. C., Golabery L., Lawson, W., Roblnson, F., Proc. Roy. Soo.. (London) B 127,140 (1939).

15. Dodds, I. C., Goldbery L., Lawson, W., Roblinson, H., Proc. Roy. $300 .$. (London) 3 127. 140 (1939).

16. Dodds, E. O., Golabery L.. Lawson, W.., Robinson, F... Proc., Roy. Soc. (London) B i27, 140 (2939).

17. Hobday, G. I, and Bhort, W. F., J. Chen. Soc. 1943 Pp 609-12.

18. Hobday, G. I. and Short, W. F., J. Cher. Boc. 1943 Pp 609-12. 


\section{ABSTRACT}

The conditions for reduction of ketones to plnacols at plated copper gauze cathodes were studied. liercury plated 40 and 60 mesh copper gauze was the most effective in both acld and alkaline nedium. Lead plated copper gauze, the second most effective cathode, was followed by cadnin, silver-mercury, thallium, nickelmeroury, and bismuth. Tin, antimony, and arsenic ala not produce the desired reaction. For acid medium $0.022, \mathrm{II}_{2} \mathrm{iO}_{4}$, as the electrolyte, eave the best results while in alkaline medium, $2 N$ Haof gave the best results. The forration of the di and meso lsomers in about erval proportions were separated by cold $20 \%$ sodium hydroxide. Both isomers undergo dehydration and acetylation to give dienestrol diacetate which, upon hydrolysis, gives dienestrol. Heating the isomers in acid causes them to undergo the pinacolone rearrangement. By the schottenBaumann reaction, the dibenzoate vias prepared and separated. 